\title{
Image analysis of the nuclear characteristics of emerin protein and the correlation with nuclear grooves and intranuclear cytoplasmic inclusions in lung adenocarcinoma
}

\author{
SAYAKA KOBAYASHI ${ }^{1}$, MASANAO SAIO ${ }^{1}$, TOSHIO FUKUDA ${ }^{1}$, \\ KIMINORI KIMURA ${ }^{2}$, JUNKO HIRATO ${ }^{3}$ and TETSUNARI OYAMA ${ }^{3,4}$
}

\begin{abstract}
${ }^{1}$ Laboratory of Histopathology and Cytopathology, Department of Laboratory Sciences, Gunma University Graduate School of Health Sciences, Gunma 371-8514; ${ }^{2}$ Department of Hepatology, Tokyo Metropolitan Cancer and Infectious Disease Center, Komagome Hospital, Tokyo 113-8677; ${ }^{3}$ Department of Pathology, Gunma University Hospital, Gunma 371-8511; ${ }^{4}$ Department of Diagnostic Pathology, Gunma University Graduate School of Medicine, Gunma 371-8511, Japan
\end{abstract}

Received August 23, 2018; Accepted October 31, 2018

DOI: $10.3892 / o r .2018 .6848$

\begin{abstract}
Nuclear size and shape are important components in the diagnosis of pathological specimens. However, a qualitative evaluation is typically applied rather than a quantitative evaluation technique. In the present study, we sought to evaluate the nuclear morphological characteristics of lung adenocarcinoma using whole-slide imaging (WSI) and computer-assisted image analysis (IA). We evaluated the nuclear characteristics of 106 cases of surgically resected lung adenocarcinoma according to Feulgen staining and immunohistochemistry (IHC) for the inner nuclear membrane protein emerin. According to the Feulgen reaction, although the nuclear area (size) of the carcinoma cells was correlated with the nuclear perimeter (NP) $(\mathrm{R}=0.8973)$, the nuclear staining intensity of carcinoma cells was not correlated with the nuclear area. Using emerin IHC, we used IA software that was able to detect both the NP and the emerin-stained nuclear membrane length (ENML) in the nucleus, and found that the more nuclei exhibited a longer ENML relative to the NP, the more nuclear grooves and intranuclear cytoplasmic inclusions were present. In addition, the nuclear area was correlated with the percentage of nuclei that had a longer ENML compared to the NP against the total nuclei $(\mathrm{R}=0.7759)$. Furthermore, the emerin low expression group showed an enlarged nuclear area $(\mathrm{P}=0.0264)$, elongated NP $(\mathrm{P}=0.0091)$, and lower shape factor $(\mathrm{P}=0.0486)$ compared with the normal emerin expression
\end{abstract}

Correspondence to: Dr Masanao Saio, Laboratory of Histopathology and Cytopathology, Department of Laboratory Sciences, Gunma University Graduate School of Health Sciences, 39-22, 3-chome, Showa-machi, Maebashi-city, Gunma 371-8514, Japan E-mail: saio@gunma-u.ac.jp

Key words: nuclear morphology, Feulgen reaction, emerin, whole slide imaging, image analysis group. Our data indicated the usefulness of WSI and IA for pathological specimen analysis. In addition, this study is the first to report that the low expression of emerin in cancer cell results in an oval shape of nuclei and nuclear enlargement in clinical samples.

\section{Introduction}

Inter-observer variance in the evaluation of pathological specimens is one of the problems that arises when pathological findings are used for experimental purposes (1). In order to resolve this, several attempts to utilize image analysis (IA) were introduced in the 1980s. For example, Stern et al and Rosenthal et al analyzed cervical samples by IA to determine the cytosolic diameter, nuclear diameter, and integrated optical density of the nucleus $(2,3)$. Since then, other attempts have been made by other groups. For example, Nakazato et al reported that cases of lung adenocarcinoma in which the cells had larger nuclei exhibited a worse prognosis than the cases in which the cells had smaller nuclei. In that study, they also showed a significant difference between cases with large nuclear size and small nuclear size with respect to pathological stage, $\mathrm{T}$ factor, $\mathrm{N}$ factor and histological classification (4). In addition to IA, whole-slide imaging (WSI) was utilized to capture digital images of the pathological specimens to generate homogeneous images. For example, Yamada et al showed the importance of nuclear analysis by IA for nuclear size, shape factor, and intra-nuclear texture features to distinguish lowgrade ductal carcinoma in situ (DCIS) from high-grade DCIS in WSI of hematoxylin and eosin (H\&E)-stained specimens of breast cancer cases (5). In addition, Kosuge et al showed the significance of diverse nuclear density and size in the evaluation of high-grade urothelial carcinomas by Feulgen staining using IA and WSI techniques (6). Thus, the effort to evaluate pathological findings in an objective manner is continuously improving.

The nuclear envelope is a structure that separates chromosomes from the cytosol in eukaryotic cells (7). Some nuclear proteins have been utilized to analyze pathological specimens. 
For example, Asioli and Bussolati reported that immunohistochemical staining for emerin, which is one of the inner nuclear membrane proteins, could predict the shapes of the nuclear membrane to help distinguish follicular variant papillary carcinoma (FVPC) from follicular tumors. In contrast, the nuclear features of FVPC were unclear in the H\&E-stained specimens (8). Bussolati et al also used lamin, another nuclear membrane protein, in addition to emerin for the analysis of the nuclear morphology of breast cancer cases (9).

In the present study, we sought to evaluate the nuclear morphology of 106 cases of lung adenocarcinoma using two types of stains. One is the Feulgen reaction, as the staining is valuable for the semi-quantitative evaluation of DNA and has a good signal to noise ratio. The other is emerin immunohistochemistry (IHC), which has been used to trace the shape of the nuclear membrane. In addition, we applied WSI and IA to analyze nuclear features in an objective manner and aimed to determine subtle differences that might otherwise be missed without these tools.

\section{Materials and methods}

Cases. We included pathological specimens from 106 patients with lung adenocarcinoma who underwent surgical resection at the Gunma University Hospital (Maebashi, Japan) from November 2011 to December 2013. Our research was approved by the Ethics Committee of Gunma University School of Medicine, and the written notification for this study was presented publicly on the webpage of our hospital. Moreover, the opportunity to decline participation in this study was guaranteed according to the Ethical Guidelines for Medical and Health Research Involving Human Subjects of the Japanese government (Ministry of Education, Culture, Sports, Science and Technology and Ministry of Health, Labour and Welfare).

Table I contains a summary of the clinical features of the samples included in this study. We classified each case based on tumor stage and tumor size according to the 2017 TNM classification of the Union for International Cancer Control (10). We also classified each case into histological categories and subcategories according to the 2015 World Health Organization Classification of Lung Tumors (11). We excluded pTla cases from this study due to the small size of these tumors.

Preparation of the specimens. Overall, 3-, 2- and 1- $\mu$ m-thick specimens were prepared from $10 \%$ formalin-fixed, paraffinembedded blocks for each staining procedure described below. Then, 3- $\mu$ m-thick sections used for H\&E staining were mounted onto Star frost glass slides (cat. no. 511511; Muto Pure Chemicals Co. Ltd., Tokyo, Japan). The specimens used for Feulgen staining and IHC were mounted onto silane-coated glass slides (NEW Silane III, cat. no. 519618; Muto Pure Chemicals Co. Ltd.).

Hematoxylin and eosin $(H \& E)$ staining. After de-paraffinization (xylene 3 times for 5 min each time) and rehydration (100\% ethanol for $1 \mathrm{~min}, 95 \%$ ethanol for $1 \mathrm{~min}$, and $70 \%$ ethanol for $1 \mathrm{~min}$ ), the specimens were rinsed in running water for $1 \mathrm{~min}$. Then, the specimens were stained with hematoxylin
Table I. Characteristics of the lung adenocarcinoma cases.

\begin{tabular}{lc}
\hline Characteristics & No. of patients \\
\hline Total number of cases & $106(100)$ \\
Age (years) & \\
$<60$ & $21(20)$ \\
$\geq 60$ & $85(80)$ \\
Sex & \\
Male & $60(57)$ \\
Female & $46(43)$ \\
pT status & \\
pTis & $1(1)$ \\
pT1 & $48(45)$ \\
pT2 & $44(42)$ \\
pT3 & $11(10)$ \\
pT4 & $2(2)$ \\
Stage & \\
I & $69(65)$ \\
II & $21(20)$ \\
III & $14(13)$ \\
IV & $2(2)$ \\
Histological subtype & \\
Adenocarcinoma in situ & $1(1)$ \\
Invasive adenocarcinoma & $99(93)$ \\
Lepidic adenocarcinoma & $30(28)$ \\
Papillary adenocarcinoma & $38(36)$ \\
Acinar adenocarcinoma & $23(22)$ \\
Micropapillary adenocarcinoma & $0(0)$ \\
Solid adenocarcinoma & $8(8)$ \\
Invasive mucinous adenocarcinoma & $6(6)$ \\
\hline
\end{tabular}

solution (New Hematoxylin Type M, cat. no. 30141; Muto Pure Chemicals) for $10 \mathrm{~min}$ at room temperature $(\mathrm{R} / \mathrm{T})$. After they were washed in running water for $10 \mathrm{~min}$, the specimens were stained with eosin solution (New Eosin Type M, cat. no. 32081; Muto Pure Chemicals) for $3 \mathrm{~min}$. The dehydration (rinse in $70 \%$ ethanol, rinse in $95 \%$ ethanol, and $100 \%$ ethanol twice for $30 \mathrm{sec}$ each time) and the penetration steps (xylene 3 times for 5 min each time) were performed. Next, the specimens were cover-slipped with mounting medium (Malinol, cat. no. 20093; Muto Pure Chemicals) for observation.

Feulgen reaction. For the Feulgen reaction, we followed the protocol reported by Kreicbergs and Zetterberg (12), except for the hydrogen chloride $(\mathrm{HCl})$ treatment time. Specifically, after the deparaffinization and hydration steps, the specimens were washed with running water for $1 \mathrm{~min}$ and distilled water (DW) for $1 \mathrm{~min}$ and were incubated with $5 \mathrm{~N} \mathrm{HCl}$ for $40 \mathrm{~min}$ in order to remove the purine bases. Then, the specimens were rinsed with cold Schiff reagent (cat. no. 40932; Muto Pure Chemicals;) once, and then stained with cold Schiff reagent for $90 \mathrm{~min}$ at $\mathrm{R} / \mathrm{T}$. In order to stop the reaction, the specimens were treated with sulfurous acid solution (cat. no. 40941; Muto Pure Chemicals) three times. After washing with running 


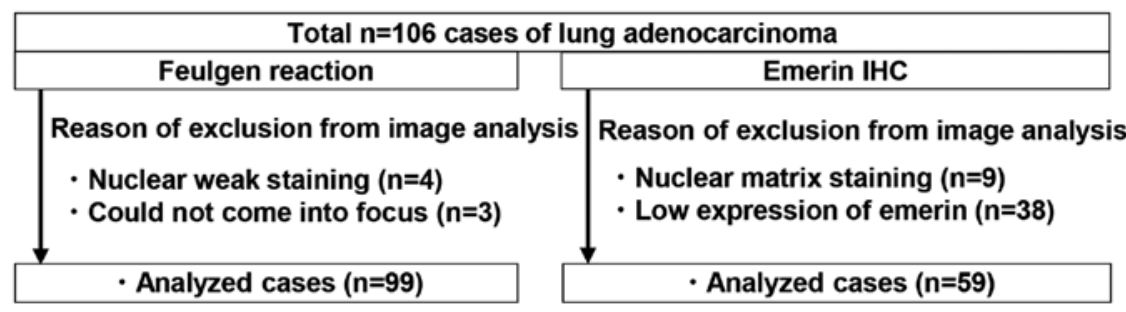

Figure 1. Flow chart of the analysis in this study shows the Feulgen reaction and emerin IHC. IHC, immunohistochemistry.

water for $5 \mathrm{~min}$, dehydration, penetration and mounting were performed.

Immunohistochemistry (IHC). After de-paraffinization and hydration, the specimens were washed in running water for $1 \mathrm{~min}$. Then, the specimens were treated with $0.3 \%$ hydrogen peroxide for $30 \mathrm{~min}$ at $\mathrm{R} / \mathrm{T}$ in order to block internal peroxidase activity. After they were washed in running water for $1 \mathrm{~min}$, the specimens were placed in a container filled with $100 \mathrm{mM}$ Tris-EDTA buffer (pH 9.0), which was then placed in a thermos containing the same buffer. The thermos was then heated at $97^{\circ} \mathrm{C}$ for $30 \mathrm{~min}$, after which the container was removed from the thermos and kept at $\mathrm{R} / \mathrm{T}$ until the specimens cooled. Then, the specimens were washed in running water and rinsed with $10 \mathrm{mM}$ phosphate-buffered saline (PBS), $\mathrm{pH}$ 7.4. To block non-specific reactions due to the secondary antibody, the specimens were treated with $2 \%$ normal goat serum for $15 \mathrm{~min}$ at $\mathrm{R} / \mathrm{T}$. After the solution was removed by tapping, the specimens were treated with mouse monoclonal anti-human emerin antibody (1:500 dilution, clone CL0201; cat. no. NBP2-52876; Novus Biologicals, Littleton, CO, USA) at $4^{\circ} \mathrm{C}$ overnight. Then, the specimens were washed with PBS 3 times for 5 min each time, and incubated with horseradish-peroxidase polymer-labeled goat anti-mouse immunoglobulin antibody (Histofine Simple Stain MAX-PO (M), cat. no. 424134; Nichirei Biosciences, Tokyo, Japan) for $30 \mathrm{~min}$ at $\mathrm{R} / \mathrm{T}$. After washing with PBS three times for 5 min each time, the reaction was visualized by treatment with $0.003 \% \mathrm{H}_{2} \mathrm{O}_{2}$ and $0.2 \mathrm{mg} / \mathrm{ml} \mathrm{3,3'-diaminobenzidine} \mathrm{(DAB)} \mathrm{in}$ $50 \mathrm{mM}$ Tris- $\mathrm{HCl}, \mathrm{pH} 7.6$, for $2 \mathrm{~min}$ at $\mathrm{R} / \mathrm{T}$. After the slides were washed in running water for $1 \mathrm{~min}$, counterstaining was performed with hematoxylin solution for $5 \mathrm{~min}$ at R/T. After another wash in running water for $5 \mathrm{~min}$, dehydration, penetration, and mounting were performed.

Whole-slide imaging (WSI). WSI of specimens subjected to the Feulgen stain and IHC were obtained by a TOCO Virtual Slide Scanner (VS) (Claro, Hirosaki, Japan) with a x40 objective lens. The specifications of the TOCO 20 are as follows: camera pixels, 1.39 million pixels; size of the pixels, $0.26 \mu \mathrm{m} /$ pixel; source of lamination, super luminosity light emitting diode. Autofocus mode was used for capture.

Image analysis. For the Feulgen stain, five randomly selected VS images (in all, more than 400 nuclei were present in each case) were saved as TIFF files. The TIFF files were converted to MRXS files using an image converter software (E-Path Co., Ltd., Kanagawa, Japan). Then, the TIFF images were analyzed by Pannoramic Viewer version 1.15.4 using the Quant Center HistoQuant module (3DHISTECK Ltd., Budapest, Hungary) for Feulgen reaction-positive areas with the following RGB range: $(200,207,230)$. In order to exclude small nuclei such as those in lymphocytes and aggregated cells, nuclei $<105 \mu \mathrm{m}^{2}$ and $>720 \mu \mathrm{m}^{2}$ were excluded from the analysis. The data collected were as follows: nuclear area, nuclear perimeter (NP), nuclear circularity, and nuclear gray intensity (staining intensity). Nuclear circularity was defined as the ratio of two concentric circles as follows: circle that lined the innermost side of the object/circle that lined the outermost side of the object. Therefore, if the area was an exact circle, the circularity was 1 . In terms of gray intensity, gray tone was indicated by 8-bit color (256). In all, 99 cases were analyzed. Several specimens were unable to be analyzed due to poor focus (4 cases) or light staining (3 cases) and were excluded from the study (Fig. 1).

In terms of the IHC specimens, five randomly selected VS images (in all, more than 200 nuclei were present in each case) were saved as TIFF files. For nuclear membrane and nuclear analyses, e-Nucle version 21 (E-path Co., Ltd., Fujisawa, Japan) was used. Data settings for analysis were as follows: red: 0-255, green: 0-255, blue: $75-102$ (1 pixel=0.26 micrometer for TOCO virtual slide scanner that we used in this study). Detected positive area size $<13 \mu \mathrm{m}^{2}$ and $>125 \mu \mathrm{m}^{2}$ was excluded from this study as lymphocytes and aggregated cells are included in these range. According to the manufacturer, 'nuclear area with DAB staining' was detected as a hematoxylin-positive area with DAB staining. First, the nuclear region that was defined by the hematoxylin-stained area was traced as the outermost part of the region and was termed NP. The circular area with positive DAB staining inside of the nuclear area was recognized as the 'emerin-stained nuclear membrane length (ENML)'. Thus, if the nuclear area was not completely surrounded by positive DAB staining, the nucleus was not detected by our protocol. On the contrary, the emerin-stained portion was detected as the linear DAB-positive portion of the 'nuclear area'. The ENML was determined by tracing the center line of emerinstained linear belt-like objects in the nuclear area. Thus, if the nucleus contained no nuclear grooves or invaginations, the NP was larger than the ENML because the NP traced the outermost part of the nucleus, whereas the ENML traced the center line of thin belt-like DAB-stained area. Using our protocol, we collected data on the nuclear area, NP, ENML, maximum diameter and minimum diameter of the nuclear area. We defined emerin low expression as cases in which less than half of tumor nuclei could be detected by our protocol. Due to nuclear matrix staining (9 cases) and weak expression 

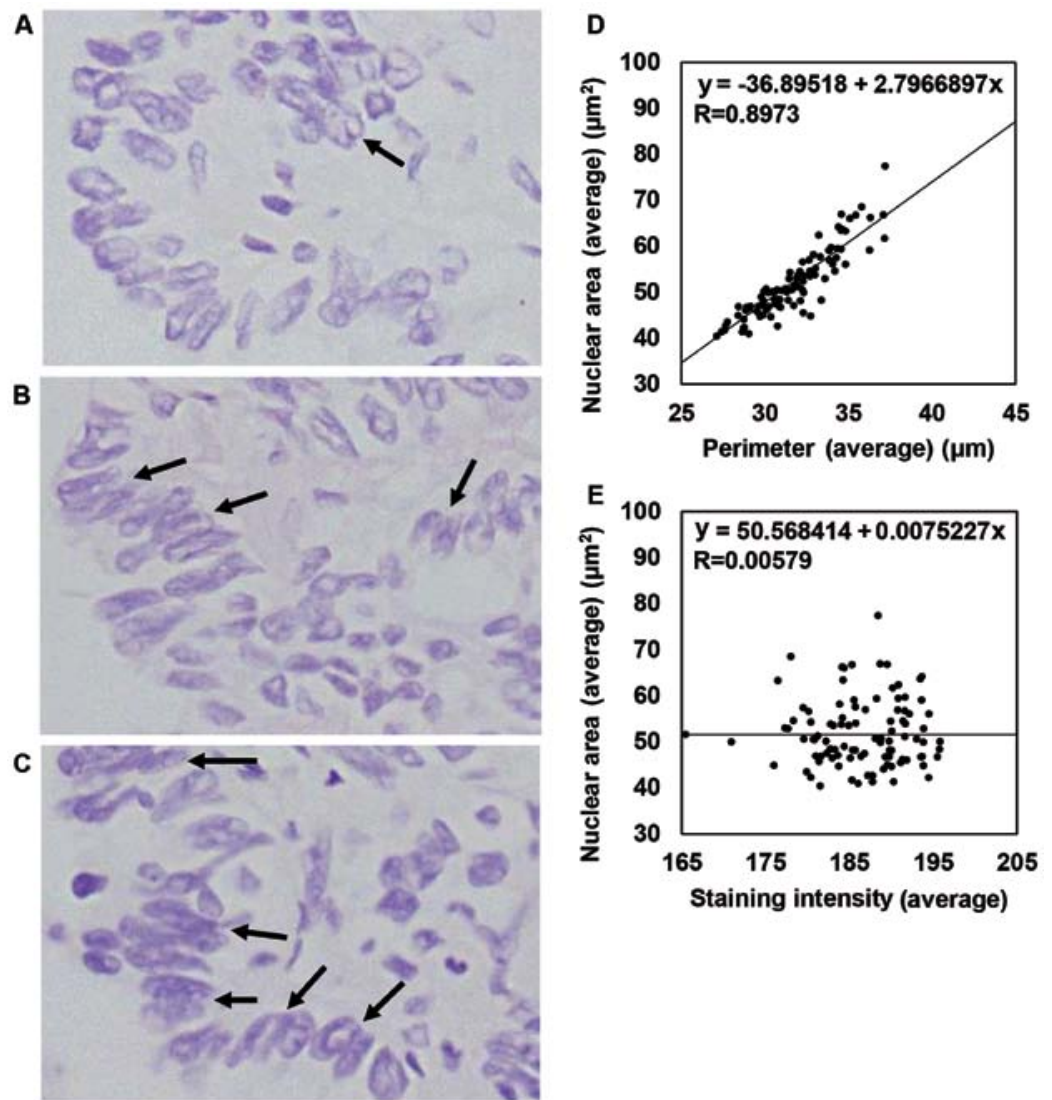

Figure 2. (A-C) Representative nuclear overlapping and the effect of specimen thickness on nuclear overlapping and (D and E) representative data from the correlation analysis for nuclear size, NP, or staining intensity of specimens stained by the Feulgen reaction. Effect of section thickness is shown in case no. 1: (A) 1- $\mu \mathrm{m}$-thick, (B) 2- $\mu \mathrm{m}$-thick and (C) 3- $\mu \mathrm{m}$-thick. (D) Correlation of nuclear area (Avg) and NP (Avg), and correlation of (E) nuclear area (Avg) and staining intensity (Avg). For A-C, images were captured using VS (x40 objective lens). NP, nuclear perimeter; VS, virtual slide scanner.

of emerin (38 cases), 47 cases were excluded from the image analysis by e-Nucle software (Fig. 1).

Statistical analysis. We utilized JMP Pro version 12.2.0 software (SAS Japan, Tokyo, Japan) for all statistical analyses. For the correlation analysis, $\mathrm{R}>0.9$ was considered to represent high correlation, whereas 0.7-0.9 indicated moderate correlation, 0.5-0.7 indicated low correlation, and 0.5 a chance result (13). When the average (Avg) was compared between the two groups, the Student's t-test was used. Results with P-values $<0.05$ were considered statistically significant.

\section{Results}

Amount of nuclear DNA did not correlate with nuclear size according to the analysis by Feulgen reaction. We utilized the Feulgen reaction for nuclear morphological analysis rather than hematoxylin staining because the Feulgen reaction has a better signal/noise ratio and allows for the analysis of DNA content in a semi-quantitative manner (6). First, we investigated the thickness of specimens that were suitable for nuclear morphological analysis in this study. We assessed $3-\mu \mathrm{m}$-thick H\&E-stained specimens and found that some exhibited nuclear overlapping (data not shown). Therefore, we prepared 1- to 3- $\mu$ m-thick sections and analyzed the nuclear morphology using our protocol. We observed representative severe nuclear overlapping in the case shown in Fig. 2A-C.
Even in cases with severe nuclear overlapping, 1- $\mu \mathrm{m}$-thick sections showed relatively minimal nuclear overlapping compared with 2- to 3- $\mu \mathrm{m}$-thick sections. Moreover, since our protocol detected the nuclear area appropriately, we chose $1-\mu \mathrm{m}$-thick sections for analysis of the Feulgen reaction. In this setting, we were able to analyze 99 cases (Fig. 1). The correlation of each factor detected in the image analysis was as follows: the Avg nuclear area versus (vs.) the standard deviation (SD) of the nuclear area $(\mathrm{R}=0.9471)$, the Avg vs. the $\mathrm{SD}$ of the NP $(\mathrm{R}=0.9002)$ demonstrated a high correlation. The Avg nuclear area vs. the Avg NP ( $\mathrm{R}=0.8973)$, the SD of the nuclear area vs. the SD of the NP $(\mathrm{R}=0.8570)$, and the SD of the NP vs. the Avg circularity ( $R=0.7167)$ demonstrated a moderate correlation. In addition, the Avg circularity vs. the $\mathrm{SD}$ of circularity ( $\mathrm{R}=0.6693)$, the Avg staining intensity vs. the $\mathrm{SD}$ of staining intensity ( $\mathrm{R}=0.7008)$ demonstrated a low correlation, but other combinations did not demonstrate any correlation. The representative results are shown in Fig. 2D and E. Our results indicate that our analysis of continuous variables provided a correlation between nuclear size and NP. In terms of staining intensity, which seemed to represent the amount of DNA in a semi-quantitative manner (6), our staining intensity was not correlated with nuclear size or morphology (Avg nuclear area, Avg NP, or Avg nuclear circularity) or with the variability in nuclear size or morphology (SD of the nuclear area, SD of the NP, or SD of the nuclear circularity). 

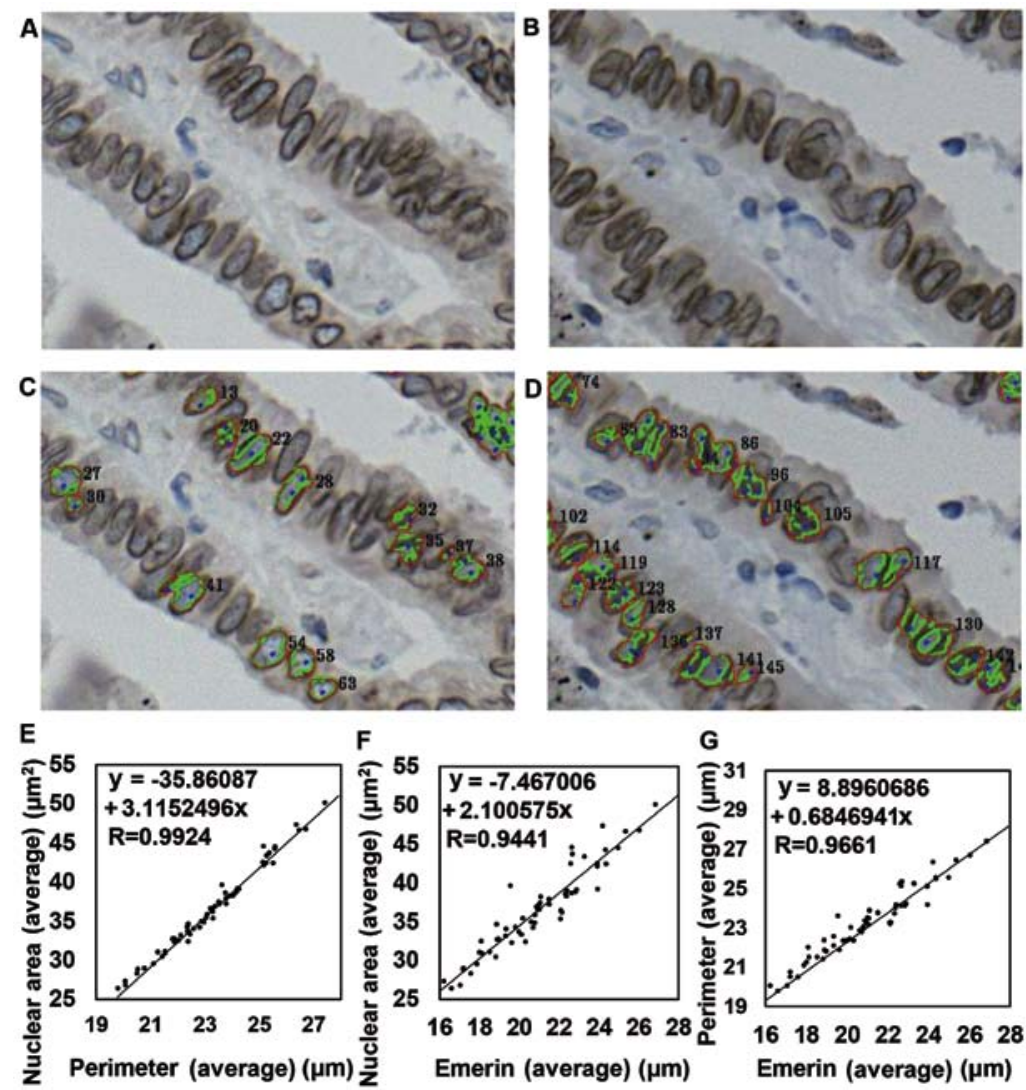

Figure 3. (A-D) Representative effect of specimen thickness in the nuclear analysis of emerin-stained specimens after image analysis and (E-G) representative data of the correlation analysis for nuclear size, NP or ENML for specimens stained by immunohistochemistry (IHC) for emerin. Effect of section thickness is shown in case no. 14: (A and B) 1- $\mu$ m-thick, (C and D) 2- $\mu$ m-thick. NP as detected by our protocol is indicated in red, while the emerin-stained membranetraced line is indicated in green (B and D). (E-G) Correlation of nuclear area (Avg), NP (Avg), and ENML. Emerin in the X-axis in (G) stands for ENML (emerin-stained nuclear membrane length). Images in A-D were captured using VS (x40 objective lens). NP, nuclear perimeter; VS, virtual slide scanner.

Nuclear morphological analysis of total nuclei in emerinstained specimens did not reflect the presence of a minor population with eccentric nuclear morphology. Since we could not properly detect the nuclear area in $1-\mu \mathrm{m}$-thick emerinstained sections using our protocol, we selected $2-\mu \mathrm{m}$-thick sections for this analysis. A representative case in which the NP and ENML could be detected in a $2-\mu$ m-thick section but one in which the NP and ENML failed to be detected in a $1-\mu$ m-thick section is shown in Fig. 3A-D. We were able to analyze 59 cases in this setting, and the correlation of each factor was as follows: the Avg nuclear area vs. the Avg of the $\mathrm{NP}(\mathrm{R}=0.9924)$, the Avg NP vs. the Avg emerin-stained nuclear membrane length (ENML) $(\mathrm{R}=0.9661)$, the Avg ENML vs. the $\mathrm{SD}$ of the nuclear area $(\mathrm{R}=0.9502)$, the Avg nuclear area vs. the Avg ENML ( $R=0.9441)$, and the SD of the NP vs. the SD of the ENML ( $R=0.9304)$ showed a strong correlation. The Avg ENML and the SD of the NP ( $R=0.8952)$, the $S D$ of the nuclear area vs. the SD of the ENML ( $\mathrm{R}=0.8863)$, the Avg nuclear area vs. the SD of the NP $(\mathrm{R}=0.7855)$ and the Avg NP vs. the $\mathrm{SD}$ of the ENML $(\mathrm{R}=0.7076)$ showed a moderate correlation. In contrast, the Avg nuclear area vs. the SD of the ENML ( $\mathrm{R}=0.6483)$ showed a weak correlation. Other combinations did not show any correlation. Representative results are shown in Fig. 3E-G. Despite the findings of nuclear grooves (NGs), intranuclear cytoplasmic inclusions (ICIs), and jagged shape of some nuclei (we termed these nuclei 'eccentric nuclei in this manuscript), the data described above suggested that nuclear morphological changes including NGs, ICIs, and jagged shape were so infrequently observed that these changes might not be exhibited if we estimated the data based on the Avg or the SD of all nuclei.

The difference in nuclear perimeter and emerin-stained nuclear membrane length accurately reflected a minor population with eccentric nuclear morphology. We next considered how we could properly reflect the existence of eccentric nuclei, nuclei with NGs, and ICI-positive nuclei, which comprised a relatively minor proportion of the total nuclei of cancer cells. We noticed that the difference in the NP minus the ENML (NP-ENML difference) would be the most appropriate way to estimate the changes in membrane morphology. To determine the NP-ENML difference, we subtracted the ENML from the NP for each nucleus examined. Then, we calculated the ratio of the NP-ENML difference in positive nuclei to the total number of nuclei. In these experiments, if the ENML was longer than the NP, we termed the nucleus as a 'high nuclear deformity nucleus (HNDN)', and we calculated the rate of HNDN out of all nuclei. We compared the NP and the presence of ICIs in cases by visual examination (i.e., with the naked eye) and accounted for the rate of HNDN. Representative cases with smooth and eccentric nuclei are shown in Fig. 4. As an average size, even in cases with eccentric nuclei, the average ENML was smaller than the average of the NP according to the software algorithm, as described in the Materials and 
A Representative smooth nuclei case

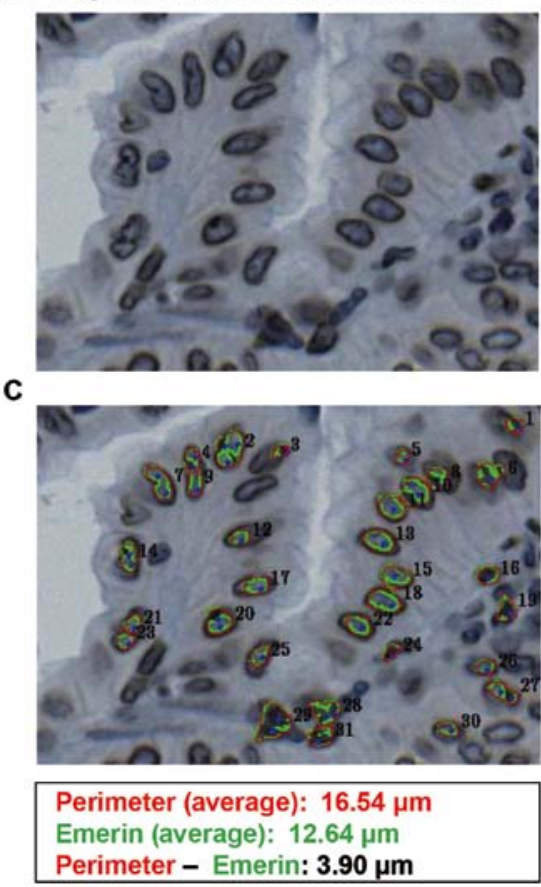

B Representative eccentric nuclear case
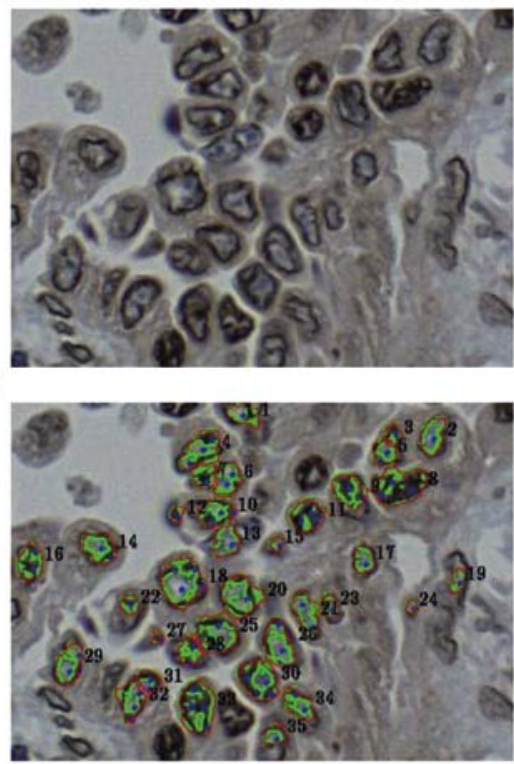

Perimeter (average): $21.79 \mu \mathrm{m}$

Emerin (average): $20.79 \mu \mathrm{m}$

Perimeter - Emerin: $1.00 \mu \mathrm{m}$

Figure 4. A representative case with predominantly smooth nuclei (case no. 24) (A and C) and a case with eccentric nuclei (case no. 97) (B and D) as shown by emerin immunohistochemistry (IHC). In B and D, the NP is indicated in red and the ENML traced by the software is shown in green. Avg data of NP and ENML (emerin) and NP minus ENML is shown at the bottom of the figure. All images were captured by a VS (x40 objective lens). NP, nuclear perimeter; ENML, emerin-stained nuclear membrane length; VS, virtual slide scanner.

methods section. As shown in Fig. 5A and B, the frequency of cases with NGs and the frequency of ICIs gradually increased based on the increase in the rate of HNDN. In addition, according to this analysis, the Avg ENML vs. the rate of HNDN ( $R=0.9256)$, the SD of the NP vs. the rate of HNDN $(\mathrm{R}=0.8975)$, and the SD of the ENML vs. the rate of HNDN $(\mathrm{R}=0.9110)$ all demonstrated a high correlation. The Avg NP vs. the rate of HNDN ( $R=0.8125)$, the Avg nuclear area and the rate of HNDN $(\mathrm{R}=0.7759)$, and the SD of the nuclear area vs. the rate of HNDN ( $R=0.8862)$ demonstrated a moderate correlation. Representative data are shown in Fig. 5C and D. These data suggested that the rate of HNDN was a valuable tool to detect cases of eccentric nuclei using objective continuous variables. In addition, our data indicated that larger nuclear size showed a tendency to be associated with a large rate of HNDN (Fig. 5E). Manual observation and evaluation of nuclear morphology against the same images used for IA were performed by two pathologists (MS and FT). The accordance rate between the two observers was $43.7 \%$, and the graphs of each observer's results appeared different from each other although the average score between the two observers demonstrated a moderate correlation $(\mathrm{R}=0.8066)$ (Fig. S1). These data indicated that manual observation and evaluation was useful but not consistent because of inter-observer variance.

The emerin low expression group showed enlarged nuclei with an oval shape compared with the emerin high expression group. Finally, we compared whether emerin expression could affect nuclear size. To explore this question, we defined the group that was excluded from the emerin immunohistochemistry (IHC) nuclear morphological analysis due to emerin low expression as the 'emerin low expression group'. Then, we compared Feulgen staining in the emerin expression group and the emerin low expression group. Among the emerin low expression group $(n=38), 34$ cases could be analyzed by Feulgen reaction. In contrast, among cases in the emerin expression group ( $\mathrm{n}=59$ ), 54 could be analyzed by Feulgen reaction. In comparison with the emerin expression group, the emerin low expression group had significantly enlarged nuclear areas $(\mathrm{P}<0.0264), \mathrm{NP}(\mathrm{P}<0.0091)$ and nuclear circularity $(\mathrm{P}<0.0486)$. However, the nuclear intensity was not significantly different between the two groups. Representative data are shown in Fig. 6. Our data indicated that low expression of emerin affects nuclear size and shape. NP, nuclear perimeter.

\section{Discussion}

High nuclear grade denotes a more aggressive tumor (14), but nuclear grade is the most subjective factor in comparison with architectural features and mitotic rate in the evaluation of breast cancer specimens (14). Therefore, we believe that the establishment of a more objective method to evaluate nuclear morphology is necessary. In the present study, we analyzed the nuclear morphology of 106 cases of lung adenocarcinoma using Feulgen staining and emerin immunohistochemistry (IHC) in combination with whole-slide imaging (WSI) and computer-assisted image analysis (IA). By the Feulgen reaction, a correlation was observed between the Avg nuclear area and the average nuclear perimeter (NP), but no correlation was observed between nuclear circularity (Avg) and nuclear area (Avg), NP (Avg), or nuclear staining intensity represented by the staining intensity (Avg) of the nucleus. In contrast, no 


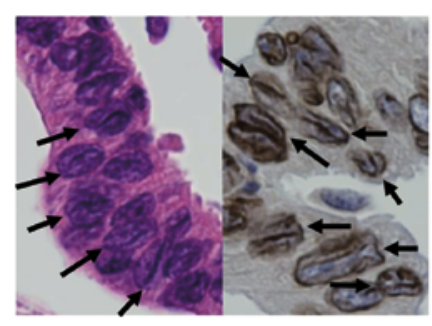

c

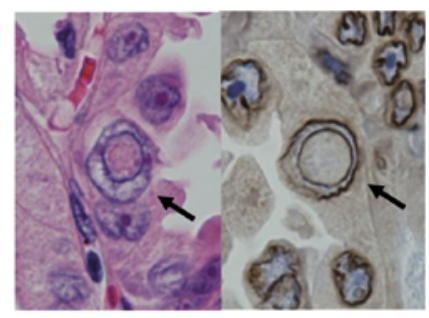

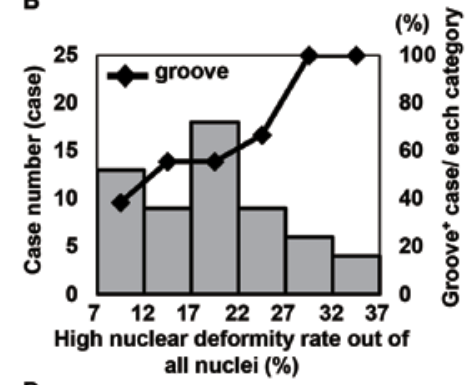

D

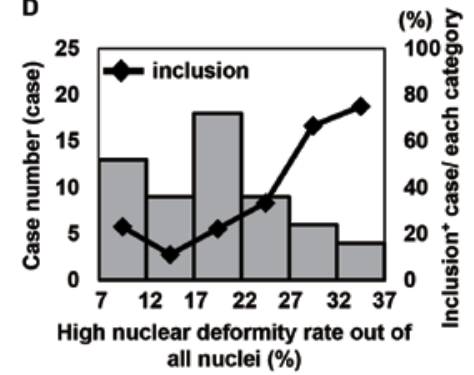

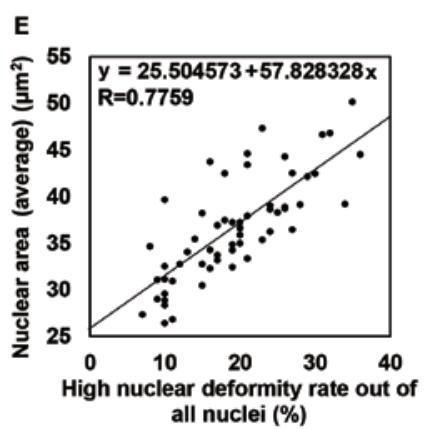

Figure 5. (A and C) Representative nuclear groove and intracytoplasmic inclusion, and (B and D) the relationship among nuclear grooves and intracytoplasmic inclusions, and a high nuclear deformity rate among all nuclei (NP minus the ENML-negative nuclei ratio out of all nuclei) in emerin-stained specimens. (E) The correlation between nuclear area and high nuclear deformity rate (HNDR) out of all nuclei. (A and C) A representative nuclear groove is seen as a 'coffee bean shape', indicated by arrows in A. A representative intracytoplasmic inclusion is indicated by an arrow in C. On the left is an H\&E-stained section, whereas the right panel shows emerin IHC (x100 objective lens). (B and D) On the x-axis, a high nuclear deformity rate out of all nuclei was categorized for every $5 \%$. The total number of cases in each category is shown in the gray bar. The percentage of cases with nuclear grooves (NGs) (B) or intranuclear cytoplasmic inclusions (ICIs) (D) out of all cases in each category is shown in the line plot. (E) Correlation between nuclear area and the rate of HNDN. NP, nuclear perimeter; ENML, emerin-stained nuclear membrane length.

correlation was found between the staining intensity of the nuclear area (Avg) and the NP (Avg). Kumar et al analyzed the nuclear morphological features of 30 cases of normal oral mucosa and well to moderately differentiated oral squamous cell carcinoma (SCC) by H\&E staining, and found that the nuclear area and the NP of SCC were significantly larger than those of normal squamous epithelium (15). According to the data of the nuclear morphological analysis of 64 cases of breast cancer, as analyzed by cytologic smear, Kashyap et al classified cellular atypia based on Robinson's grading system. They also found that cases of high-grade cellular atypia had enlarged nuclear areas and NPs, but neither nuclear circularity, nuclear irregularity, nor nuclear staining intensity was statistically different with respect to tumor grade (16). These data suggested that nuclear enlargement does not influence circularity or staining intensity of the nucleus, and our results were consistent with this finding. In terms of staining intensity detected by the Fuelgen reaction, the reaction was reported to be utilized to evaluate DNA content of cells in a specimen in a semi-quantitative manner (6). Biesterfeld et al reported that Feulgen staining remains the gold standard for precise analysis of DNA ploidy using image cytometry (17). However, our data did not show any correlation between staining intensity and other nuclear factors. One possible explanation is the thickness of specimens. Kosuge et al utilized 3- $\mu$ m-thick specimens for analysis (6), whereas we used $1-\mu \mathrm{m}$-thick specimens. Thus it is possible that evaluation of $1-\mu \mathrm{m}$-thick specimen might be out of range of evaluation by the Feulgen reaction.

We will now discuss the relationship among nuclear grooves (NGs), cytoplasmic inclusions (ICIs) and nuclear irregularity as detected by emerin IHC in this study. Currently, the frequency of NGs and ICIs in renal cell carcinoma histological specimens is 96 and 65\%, respectively (18). In contrast, the frequency of ICIs in cytological specimens of ovarian cancer is $38 \%(19)$, while the frequency of ICIs in cytological specimens of thyroid papillary carcinoma cases is $88.9 \%$ (20). In terms of lung adenocarcinoma cases, Choi et al reported the frequency of either NGs or ICIs in anaplastic lymphoma kinase (ALK)-positive cases and ALK-negative cases as 21.1 and 9.9\%, respectively (21). In the present study, the frequency of NGs and ICIs in our cases was 61 and 31\%, respectively. That is, the frequency of NGs and ICIs was very high in our case compared to that in Choi's report. We therefore considered the reason for the high detection rate in our study. In Choi's study, H\&E-stained specimens 

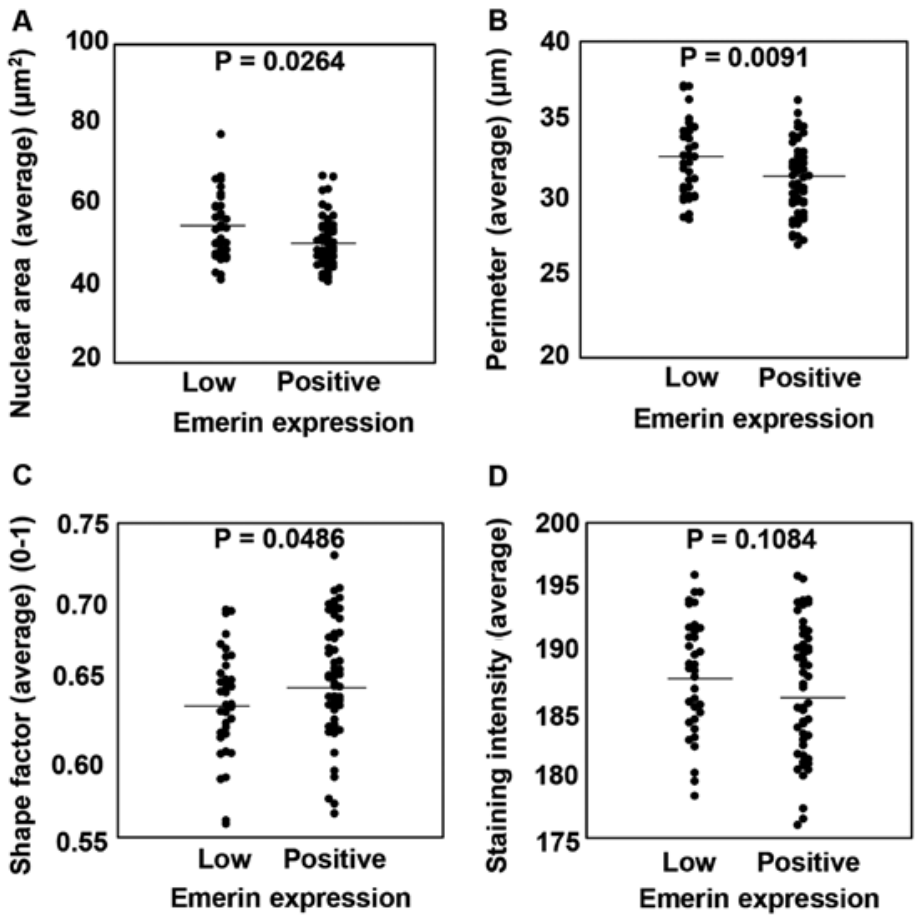

Figure 6. Comparison of cases with low emerin expression $(n=34)$ and cases with positive emerin expression ( $n=54)$. (A) Nuclear area, (B) NP, (C) shape factor, and (D) staining intensity of each group were analyzed by Student's t-test. NP, nuclear perimeter.

were examined, while in the present study, emerin-stained specimens were used for the evaluation of NGs and ICIs. Therefore, the type of staining might affect the detection rate of NGs and ICIs. Indeed, Asioli and Bussolati reported that emerin IHC allowed for easier observation of nuclear morphological structure than H\&E staining as in some H\&E-stained specimens, overstaining of the nucleus by hematoxylin led to difficulties in the observation of nuclear details (8).

Now, we will discuss the meaning of the subtraction of the emerin-stained nuclear membrane length (ENML) from the NP. In our protocol used in this study, the NP was recognized as the outermost part of the nuclear area, whereas the ENML traced the center line of the linearly stained emerin-positive membrane structure, as described in the Materials and methods section. Thus, the length of the center line of the emerinstained linear membrane structure would be smaller than the outermost part of the nuclear area if the nucleus was round without any nuclear invaginations, NGs, or ICIs. Therefore, if the subtracted value of 'NP - ENML' is negative, the nucleus would have a great number of nuclear structural changes, such as NGs or ICIs. Thus, according to our data, the frequency of NGs or ICIs was correlated with the frequency of a high nuclear deformity rate (HNDN) (Fig. 5A and B). In addition, a rate of HNDN was correlated with nuclear size (Fig. 5C). This result suggested that nuclear size is a very important factor for the occurrence of nuclear irregularities. However, no direct evidence was found to support our hypothesis, and thus, this would be a question to be resolved in a future study.

Finally, we discuss the meaning of low emerin expression and nuclear morphology. Emerin is a small $29-\mathrm{kDa}$ inner nuclear membrane protein with a single transmembrane domain that plays multiple roles (22). For example, emerin contributes to miRNA expression and myogenic signaling including that mediated by the Notch, Wnt, TGF- $\beta$ and IGF pathways (23). Additionally, emerin plays several roles in nuclear architecture since it binds directly to both type A and type $\mathrm{B}$ lamins, which are type $\mathrm{V}$ intermediate filament proteins that maintain nuclear laminar structure (24). In the present study, we found that the emerin low expression group showed larger nuclear size and NP and was more likely to have an oval shape in comparison with the emerin expression group, although the nuclear DNA content evaluated by staining intensity of the nucleus after Feulgen staining did not reveal any significant differences. In terms of the relationship between nuclear morphology and emerin expression in clinical samples, Jieying et al reported that the nuclei of emerin-expressing non-tumor follicular cells of thyroid glands exhibited a round nuclear shape and small nuclear size with a thyroxin (T4)- and thyroglobulin (Tg)-positive functional phenotype. However, the nuclei in emerin-negative cases exhibited an oval shape and large size with a T4-negative, Tg-negative dysfunctional phenotype (25).

Our data and the data of others suggested that low emerin expression results in an oval nuclear shape and large nuclear size. However, it is possible that nuclear morphological changes might result in the low expression of emerin. We further searched the literature and found two articles that described nuclear morphological changes when emerin expression was knocked down. Smith et al examined nuclear morphological changes in murine embryonic stem (ES) cells during differentiation induced by retinoic acid (RA). They found that the shape of the nuclei remained oval in wild-type (wt) cells and in single or double knockout ES cells in which the nuclear lamin $\mathrm{A} / \mathrm{C}$ proteins or emerin expression were knocked down without RA-induced differentiation. However, the nuclei became round in wt cells with RA-induced differentiation, while the shape 
of the nuclei remained oval in single or double knockouts of lamin A/C or emerin with RA-induced differentiation (26). These data indicate that lamin $\mathrm{A} / \mathrm{C}$ or emerin might contribute to a round nuclear shape during the differentiation process. Lammerding et al examined morphological features in emerindeficient murine ES cells and showed that emerin-deficient cells had an irregular nuclear shape, low contour ratio $[4 \pi \mathrm{x}$ nuclear area/(perimeter of nucleus) $\left.{ }^{2}\right]$, and enlarged nuclear size compared with wt cells (27). These data suggested that emerin downregulation itself might lead to an oval nuclear shape and enlarged nuclear size. Therefore, we believe that the changes in nuclear shape and size observed in cases with low emerin expression in our study would be directly caused by emerin downregulation. This is the first description that low emerin expression would contribute to an oval nuclear shape and enlarged nuclear size in adenocarcinoma cells in clinical samples.

In conclusion, in the present study, it was shown that a portion of cancer cell nuclei exhibited strong morphological changes, which are very difficult to evaluate with the naked eye, and thus, we used WSI and IA. In addition, our data revealed that low expression of emerin contributes not only to the oval shape of nuclei, but also to the enlargement in nuclear size.

\section{Acknowledgements}

Not applicable.

\section{Funding}

Research funding from Gunma University for MS and SK was used in these experiments.

\section{Availability of data and materials}

The datasets used and/or analyzed during the current study are available from the corresponding author on reasonable request.

\section{Authors' contributions}

SK conducted the clinical data collection, specimen staining, digital imaging of the specimens by virtual slide scanner, image analysis, statistical analysis, figure preparation, and manuscript preparation. MS developed the experimental design, conducted the experiments, digital imaging of specimens by virtual slide scanner, image analysis, figure preparation, statistical analysis and manuscript preparation. KK assisted in image analysis and manuscript reviewing for manuscript preparation. TF performed manual evaluation of emerin-stained IHC specimens, assisted in the pathological review of the cases, and reviewed and prepared the manuscript. $\mathrm{JH}$ and TO assisted in pathological review of the cases and manuscript reviewing for manuscript preparation. All authors read and approved the manuscript and agree to be accountable for all aspects of the research in ensuring that the accuracy or integrity of any part of the work are appropriately investigated and resolved.

\section{Ethics approval and consent to participate}

Our research was approved by the Ethical Committee of Gunma University School of Medicine, and the written notification for this study including notification of possibility to publish the results of this study was presented publicly on the webpage of our hospital as of information disclosure document. Moreover, the opportunity to decline participation in this study was guaranteed according to the Ethical Guidelines for Medical and Health Research Involving Human Subjects of the Japanese government (Ministry of Education, Culture, Sports, Science and Technology and Ministry of Health, Labour and Welfare).

\section{Patient consent for publication}

Not applicable.

\section{Competing interests}

The authors declare that they have no competing interests.

\section{References}

1. Nakazato Y, Maeshima AM, Ishikawa Y, Yatabe Y, Fukuoka J, Yokose T, Tomita Y, Minami Y, Asamura H, Tachibana K, et al: Interobserver agreement in the nuclear grading of primary pulmonary adenocarcinoma. J Thorac Oncol 8: 736-743, 2013.

2. Stern E, Rosenthal DL, McLatchie C, White BS and Castleman KR: An expanded cervical cell classification system validated by automated measurements. Anal Quant Cytol 4: 110-114, 1982.

3. Rosenthal DL, McLatchie C, Stern E, White BS and Castleman KR: Endocervical columnar cell atypia coincident with cervical neoplasia characterized by digital image analysis. Acta Cytol 26: 115-120, 1982.

4. Nakazato Y, Minami Y, Kobayashi H, Satomi K, Anami Y, Tsuta K, Tanaka R, Okada M, Goya T and Noguchi M: Nuclear grading of primary pulmonary adenocarcinomas: Correlation between nuclear size and prognosis. Cancer 116: 2011-2019, 2010

5. Yamada M, Saito A, Yamamoto Y, Cosatto E, Kurata A, Nagao T, Tateishi A and Kuroda M: Quantitative nucleic features are effective for discrimination of intraductal proliferative lesions of the breast. J Pathol Inform 7: 1, 2016.

6. Kosuge N, Saio M, Matsumoto H, Aoyama H, Matsuzaki A and Yoshimi N: Nuclear features of infiltrating urothelial carcinoma are distinguished from low-grade noninvasive papillary urothelial carcinoma by image analysis. Oncol Lett 14: 2715-2722, 2017.

7. Webster M, Witkin KL and Cohen-Fix O: Sizing up the nucleus: Nuclear shape, size and nuclear-envelope assembly. J Cell Sci 122: 1477-1486, 2009.

8. Asioli S and Bussolati G: Emerin immunohistochemistry reveals diagnostic features of nuclear membrane arrangement in thyroid lesions. Histopathology 54: 571-579, 2009.

9. Bussolati G, Marchiò C, Gaetano L, Lupo R and Sapino A: Pleomorphism of the nuclear envelope in breast cancer: A new approach to an old problem. J Cell Mol Med 12: 209-218, 2008.

10. O'Sullivan B, Mason M, Asmura H, Lee A, Van Eychen E, Denny L, MB A and Gupta S: Lung. In: TNM Classification of Malignant Tumours, Eighth Edition. Brierley JD, Gospodarowicz MK and Wittekind C (eds). Wiley Blackwell, West Sussex, pp106-112, 2017.

11. Travis W, Ladanyi M, Scagliotti G, Noguchi M, Meyerson M, Thunnissen E, Yatabe Y, Mino-Kenudson M, To K, Brambilla E, et al: Adenocarcinoma. In: WHO Classification of Tumours of the Lung, Pleura, Thymus and Heart. Travis W, Brambilla E, Burke A, Marx A and Nicholson A (eds). International Agency for Research on Cancer, Lyon, pp26-37, 2015.

12. Kreicbergs A and Zetterberg A: Cytophotometric DNA measurements of chondrosarcoma: Methodologic aspects of measurements in tissue sections from old paraffin-embedded specimens. Anal Quant Cytol 2: 84-92, 1980.

13. Akobeng AK: Understanding diagnostic tests 3: Receiver operating characteristic curves. Acta Paediatr 96: 644-647, 2007.

14. Fischer A: The diagnostic pathology of the nuclear envelope in human cancers. In: Cancer Biology and the Nuclear Envelope. Schirmer EC and de las Heras JI (eds). Springer, London, pp49-75, 2014. 
15. Kumar M, Chatterjee K, Purkait SK and Samaddar D: Computerassisted morphometric image analysis of cells of normal oral epithelium and oral squamous cell carcinoma. J Oral Maxillofac Pathol 21: 24-29, 2017.

16. Kashyap A, Jain M, Shukla S and Andley M: Role of nuclear morphometry in breast cancer and its correlation with cytomorphological grading of breast cancer: A study of 64 cases. J Cytol 35: 41-45, 2018

17. Biesterfeld S, Beckers S, Del Carmen Villa Cadenas $M$ and Schramm M: Feulgen staining remains the gold standard for precise DNA image cytometry. Anticancer Res 31: 53-58, 2011.

18. Lee JH, Han EM, Lin ZH, Wu ZS, Lee ES and Kim YS: Clinicopathologic significance of nuclear grooves and inclusions in renal cell carcinoma: Image database construction and quantitative scoring. Arch Pathol Lab Med 132: 940-946, 2008.

19. Naka M, Ohishi Y, Kaku T, Watanabe S, Tamiya S, Ookubo F, Kato K, Oda Y and Sugishima S: Identification of intranuclear inclusions is useful for the cytological diagnosis of ovarian clear cell carcinoma. Diagn Cytopathol 43: 879-884, 2015.

20. Das DK: Intranuclear cytoplasmic inclusions in fine-needle aspiration smears of papillary thyroid carcinoma: A study of its morphological forms, association with nuclear grooves, and mode of formation. Diagn Cytopathol 32: 264-268, 2005.

21. Choi IH, Kim DW, Ha SY, Choi YL, Lee HJ and Han J: Analysis of histologic features suspecting anaplastic lymphoma kinase (ALK)-expressing pulmonary adenocarcinoma. J Pathol Transl Med 49: 310-317, 2015.
22. Koch AJ and Holaska JM: Emerin in health and disease. Semin Cell Dev Biol 29: 95-106, 2014.

23. Koch AJ and Holaska JM: Loss of emerin alters myogenic signaling and miRNA expression in mouse myogenic progenitors. PLoS One 7: e37262, 2012.

24. Holaska JM and Wilson KL: Multiple roles for emerin: Implications for Emery-Dreifuss muscular dystrophy. Anat Rec A Discov Mol Cell Evol Biol 288: 676-680, 2006.

25. Jieying W, Kondo T, Yamane T, Nakazawa T, Oishi N, Kawasaki T, Mochizuki K, Dongfeng N and Katoh R: Heterogeneous immunoreactivity of emerin, a nuclear envelope LEM-domain protein, in normal thyroid follicles. Acta Histochem Cytochem 47: 289-294, 2014

26. Smith ER, Meng Y, Moore R, Tse JD, Xu AG and Xu XX: Nuclear envelope structural proteins facilitate nuclear shape changes accompanying embryonic differentiation and fidelity of gene expression. BMC Cell Biol 18: 8, 2017.

27. Lammerding J, Hsiao J, Schulze PC, Kozlov S, Stewart CL and Lee RT: Abnormal nuclear shape and impaired mechanotransduction in emerin-deficient cells. J Cell Biol 170: 781-791, 2005.

This work is licensed under a Creative Commons

Attribution-NonCommercial-NoDerivatives 4.0 International (CC BY-NC-ND 4.0) License. 